\title{
Multi-jet merged top-pair production including electroweak corrections
}

\author{
Christian Gütschow $^{1}$, Jonas M. Lindert ${ }^{2, a}$, Marek Schönherr ${ }^{3}$ \\ ${ }^{1}$ Department of Physics and Astronomy, University College London, Gower Street, London WC1E 6BT, UK \\ ${ }^{2}$ Department of Physics, Institute for Particle Physics Phenomenology, Durham University, Durham DH1 3LE, UK \\ 3 Theoretical Physics Department, CERN, 1211 Geneva 23, Switzerland
}

Received: 15 March 2018 / Accepted: 11 April 2018 / Published online: 19 April 2018

(C) The Author(s) 2018

\begin{abstract}
We present theoretical predictions for the production of top-quark pairs in association with jets at the LHC including electroweak (EW) corrections. First, we present and compare differential predictions at the fixed-order level for $t \bar{t}$ and $t \bar{t}+$ jet production at the LHC considering the dominant NLO EW corrections of order $\mathcal{O}\left(\alpha_{\mathrm{s}}^{2} \alpha\right)$ and $\mathcal{O}\left(\alpha_{\mathrm{s}}^{3} \alpha\right)$ respectively together with all additional subleading Born and one-loop contributions. The NLO EW corrections are enhanced at large energies and in particular alter the shape of the top transverse momentum distribution, whose reliable modelling is crucial for many searches for new physics at the energy frontier. Based on the fixed-order results we motivate an approximation of the EW corrections valid at the percent level, that allows us to readily incorporate the EW corrections in the MEPs@NLO framework of SHERPA combined with OPENLOOPS. Subsequently, we present multi-jet merged parton-level predictions for inclusive top-pair production incorporating NLO QCD + EW corrections to $t \bar{t}$ and $t \bar{t}+$ jet. Finally, we compare at the particle-level against a recent $8 \mathrm{TeV}$ measurement of the top transverse momentum distribution performed by ATLAS in the lepton + jet channel. We find very good agreement between the Monte Carlo prediction and the data when the EW corrections are included.
\end{abstract}

\section{Introduction}

The study of top-quark production and decay plays a key role in the ongoing physics programme of the LHC. Measurements in the different production modes can be used for detailed exploration of top-quark interactions as well as properties such as the top-quark mass, which is one of the fundamental parameters of the Standard Model (SM). At the same time, top-quark production and in particular top-quark pair

a e-mail: jonas.lindert@gmail.com production represents an important and challenging background in many searches for physics beyond the Standard Model (BSM). The sensitivity of many of these searches depends in a critical way on the precision of theoretical simulations in particular in the tails of kinematic distributions.

Theoretical predictions for $t \bar{t}$ production (in association with jets) at hadron colliders are indeed very advanced. For on-shell top-quarks higher-order corrections have been calculated fully differentially up to NNLO in the strong coupling [1,2] and up to NLO in the EW coupling [3-8]. These calculations have also been combined within a joint setup $[9,10]$. In fact, here also the subleading one-loop corrections, first considered in [8], have been included. Considering $t \bar{t}$ production in association with additional jets, for $t \bar{t}+$ jet $[11,12], t \bar{t}+2$ jets [13-17] and even $t \bar{t}+3$ jets [18] higher-order corrections are known at NLO QCD reducing the otherwise significant theoretical uncertainties in the modelling of top-pair plus multi-jet signatures, relevant for $t \bar{t} H$ and new-physics searches. Beyond the on-shell approximation, NLO QCD corrections for combined $t \bar{t}$ production and decay have first been studied in the narrow-width-approximation (NWA) $[6,19,20]$ and later also fully off-shell based on the $W^{+} W^{-} b \bar{b}$ final-state with off-shell leptonic [21-25] and semi-leptonic [26] decays. Including off-shell leptonic decays also the NLO EW corrections are known [27]. Furthermore, NLO QCD corrections to top-pair production in association with an additional jet combining corrections in production and decay have been computed in the NWA [28] and also fully off-shell with leptonic decays [29]. Finally, in $[8,9]$ the photon-initiated production of top-quark pairs has been studied.

Particle-level Monte Carlo generators matching NLO QCD matrix elements to parton showers have been available for quite some time for inclusive $t \bar{t}$ production $[30,31]$ and since more recently for $t \bar{t}+$ jet [32-34] and $t \bar{t}+2$ jet [35-40] production. In fact, in [38] a unified description of top-pair plus 
multi-jet production has been presented merging $t \bar{t}+0,1$, 2 jets at NLO QCD within the MEPs@NLOframework of SHERPA+OPENLOOPS [41-43]. The NLO QCD computation for combined top-pair production and decay in the NWA has been matched to parton showers [44], and the matching of fully off-shell NLO QCD top-pair production including leptonic decays was presented in [45]. The latter required the development of a modified resonance-aware NLO matching scheme [46].

Experimental cross-section measurements for top-pair production at the LHC are similarly advanced. After initial measurement at the inclusive cross section level [4752], where very good agreement with perturbative calculations at the NNLO + NNLL level in QCD $[1,53,54]$ has been observed, the attention in the study of top-pair production has shifted towards detailed differential measurements, see e.g. [55-63]. One of the most important observables in $t \bar{t}$ production, in particular relevant for beyond the Standard Model searches at the energy frontier, is the transverse momentum distribution of (reconstructed) top quarks. Different measurements of this observables consistently indicate that the top quark transverse momentum distribution at low $p_{\mathrm{T}}$ is well predicted by the employed Monte Carlo programs, both in normalisation and shape, but these predictions exceed the data at high $p_{\mathrm{T}}$. Comparing these measurements at the unfolded parton level to differential NNLO QCD predictions [2], this excess has been alleviated. This indicates the relevance of including higher jet multiplicities for the modelling of the top transverse momentum spectrum at large $p_{\mathrm{T}}$. At the same time at large $p_{\mathrm{T}}$ the higher-order EW corrections are enhanced due to the appearance of EW Sudakov logarithms $[3-8,27]$ yielding shape distortions at the level of $-10 \%$ for $p_{\mathrm{T}, \mathrm{top}}=1 \mathrm{TeV}$.

In this paper we present multi-jet merged predictions for top-pair production including QCD and EW corrections. To this end, we first present the original calculation of $t \bar{t}+$ jet production at NLO EW, i.e. the corrections of $\mathcal{O}\left(\alpha_{\mathrm{s}}^{3} \alpha\right)$, and we also consider the subleading one-loop corrections of $\mathcal{O}\left(\alpha_{\mathrm{s}}^{2} \alpha^{2}\right)$ and $\mathcal{O}\left(\alpha_{\mathrm{s}} \alpha^{3}\right)$. We compare these corrections with the corresponding ones for inclusive $t \bar{t}$ production. This comparison allows to estimate non-factorising NNLO mixed QCD-EW contributions to $t \bar{t}$ production. Furthermore, we present predictions merging $t \bar{t}$ and $t \bar{t}+$ jet production at NLO QCD + EW within the MEPs@NLO multi-jet merging framework in SHERPA combined wit OPENLOOPS, incorporating the EW corrections in an approximation [64] that we show holds at the one percent level. In this approximation, the dominant virtual NLO EW corrections are incorporated exactly, while the NLO QED bremsstrahlung is first integrated out and subsequently incorporated via YFS multi-photon emission [65]. Finally, we compare the resulting MEPs@NLOQCD + EW virt predictions, including spin-correlation preserving top-quark decays at LO, for the reconstructed top-quark transverse momentum distribution at particle-level against a recent measurement performed by ATLAS in the lepton + jet channel at $8 \mathrm{TeV}$, based on a selection of top-quark candidates in the boosted regime [59]. We find very good agreement between the Monte Carlo prediction and the data when the EW corrections are included.

The structure of this paper is as follows. In Sect. 2 we present fixed-order predictions for $t \bar{t}$ and $t \bar{t}+$ jet production including all one-loop electroweak corrections. In Sect. 3 we present the methodology of incorporating the NLO EW corrections in the MEPs@NLOframework. Resulting predictions at parton- and particle-level merging the zero and one jet multiplicties at NLO QCD + EW are presented in Sect. 4 . We conclude in Sect. 5.

\section{Electroweak corrections for $p p \rightarrow t \bar{t}+0,1$ jet}

In the following we present electroweak corrections to the processes

$p p \rightarrow t \bar{t}$ and $p p \rightarrow t \bar{t}+$ jet

which are described at leading order (LO) at $\mathcal{O}\left(\alpha_{\mathrm{s}}^{2}\right)$ and $\mathcal{O}\left(\alpha_{\mathrm{s}}^{3}\right)$, respectively. Additionally, there are subleading Born contributions of $\mathcal{O}\left(\alpha_{\mathrm{s}} \alpha\right)$ and $\mathcal{O}\left(\alpha^{2}\right)$ to $p p \rightarrow t \bar{t}$ production and of $\mathcal{O}\left(\alpha_{\mathrm{s}}^{2} \alpha\right)$ and $\mathcal{O}\left(\alpha_{\mathrm{s}} \alpha^{2}\right)$ to $p p \rightarrow t \bar{t}+$ jet production. ${ }^{1}$ The $\mathcal{O}\left(\alpha_{\mathrm{s}} \alpha\right)$ contribution to $t \bar{t}$ production is strongly suppressed as only $b$-quark-initiated processes contribute where diagrams involving a $t$-channel $W^{ \pm}$exchange interfere with diagrams involving an $s$-channel gluon. All other contributions vanish due to their colour structure. In $t \bar{t}+$ jet production all $q \bar{q}$ channels contribute at both subleading Born orders.

At the one-loop level the electroweak corrections comprise $\mathcal{O}\left(\alpha_{\mathrm{s}}^{2} \alpha\right), \mathcal{O}\left(\alpha_{\mathrm{s}} \alpha^{2}\right)$ and $\mathcal{O}\left(\alpha^{3}\right)$ contributions to $t \bar{t}$ production, and $\mathcal{O}\left(\alpha_{\mathrm{s}}^{3} \alpha\right), \mathcal{O}\left(\alpha_{\mathrm{s}}^{2} \alpha^{2}\right)$ and $\mathcal{O}\left(\alpha_{\mathrm{s}} \alpha^{3}\right)$ contributions to $t \bar{t}+$ jet production. Customarily, the leading one-loop electroweak contributions are denoted as NLO EW corrections, i.e. the relative corrections of $\mathcal{O}(\alpha)$ with respect to the LO processes. At large energies these contributions develop a logarithmic enhancement that factorises from the respective LO contributions [67-69].

In order to introduce the notation, we define the components of a $\mathcal{O}\left(\alpha_{\mathrm{s}}^{i} \alpha^{j}\right)$ Born-level computation as

$\mathrm{d} \sigma_{i j}^{\mathrm{LO}}=\mathrm{d} \Phi_{B} \mathrm{~B}_{i j}\left(\Phi_{B}\right)$

\footnotetext{
1 In our calculation we do not consider photon induced processes. As shown in [9] these contribute only at the one percent level for $t \vec{t}$ production given PDFs based on the LUXqed methodology [66] are used. We verified that this also holds for $t \bar{t}+$ jet production, where relative contributions of photon-induced production are even smaller compared to $t \bar{t}$ production.
} 
Therein, $\mathrm{B}_{i j}$ is the $\mathcal{O}\left(\alpha_{\mathrm{s}}^{i} \alpha^{j}\right)$ matrix element including all PDF and symmetry/averaging factors and $\mathrm{d} \Phi_{B}$ is its accompanying phase-space configuration. As a shorthand notation corresponding predictions are denoted as $\mathrm{LO}_{i j}$. Correspondingly, we define a one-loop correction of $\mathcal{O}\left(\alpha_{\mathrm{s}}^{i} \alpha^{j}\right)$ as

$\mathrm{d} \sigma_{i j}^{\Delta \mathrm{NLO}}=\mathrm{d} \Phi_{B} \tilde{\mathrm{V}}_{i j}\left(\Phi_{B}\right)+\mathrm{d} \Phi_{R} \mathrm{R}_{i j}\left(\Phi_{R}\right)$.

Here, $\mathrm{R}_{\mathrm{ij}}$ and $\mathrm{d} \Phi_{R}$ denote the corresponding real-emission matrix element and phase space, respectively, while $\tilde{V}_{i j}$ contains the virtual correction $\mathrm{V}_{i j}$ as well as the collinear counterterm of the PDF mass factorisation. Again as a short-hand notation these one-loop corrections are denoted as $\Delta \mathrm{NLO}_{i j}$.

All these contributions to $t \bar{t}$ and $t \bar{t}+$ jet production can readily be computed within the SHERPA+OPENLOOPS framework. ${ }^{2}$ In this framework the virtual corrections are computed with the OPENLOOPS amplitude provider [70-72], which implements a very fast hybrid tree-loop recursion to construct and compute one-loop scattering amplitudes in the full SM. For the integral reduction OPENLOOPS is interfaced with COLLIER [73] and CUTTOOLs [74]. The tree-level matrix elements as well as the infrared subtraction, process management and phase-space integration of all contributing partonic channels, are provided by SHERPA through its tree-level matrix element generator AMEGIC [75]. In SHERPA, infrared divergences are subtracted using a generalisation of the Catani-Seymour scheme [76-81], used previously in $[64,72,82-85]$, and include the appropriate initial state mass factorisation counter terms. Cross-checks of the renormalised pole coefficients of the virtual corrections computed by OPENLOOPS and the infrared poles supplied by SHERPA have been performed and excellent agreement has been found.

For our predictions of $t \bar{t}$ and $t \bar{t}+$ jet production we choose input parameters in accordance with Table 1. The electroweak coupling $\alpha$ is fixed and renormalised according to the $G_{\mu}$-scheme, $\alpha=\frac{\sqrt{2}}{\pi} G_{\mu}\left|\mu_{W}^{2} \sin _{\theta_{\mathrm{w}}}^{2}\right|$, guaranteeing an optimal description of pure $\mathrm{SU}(2)$ interactions at the EW scale. Here, $\mu_{W}$ denotes the complex-valued $W$ mass, with $\mu_{V}^{2}=m_{V}^{2}-i \Gamma_{V} m_{V}$ and $\theta_{\mathrm{w}}$ the equally complex valued weak mixing angle, derived from the ratio $\mu_{W} / \mu_{Z}$. The massive vector bosons and the Higgs are renormalised in the complexmass scheme [86], while the top-quark is kept stable and correspondingly renormalised in the on-shell scheme. The introduction of finite widths for the massive vector bosons is mandatory due to the appearance of otherwise singular resonant internal propagators in the bremsstrahlung to $t \bar{t}+$ jet production. As renormalisation and factorisation scales for the strong coupling $\alpha_{\mathrm{s}}$ we use

\footnotetext{
2 The extension of these tools to provide higher-order electroweak corrections will very soon be publicly released.
}

Table 1 Numerical values of input parameters. While the masses are taken from [88], the widths are obtained from state-of-the-art calculations

$G_{\mu}=1.1663787 \times 10^{-5} \mathrm{GeV}^{2}$

$m_{W}=80.385 \mathrm{GeV}$

$\Gamma_{W}=2.0897 \mathrm{GeV}$

$m_{Z}=91.1876 \mathrm{GeV}$

$\Gamma_{Z}=2.4955 \mathrm{GeV}$

$m_{h}=125 \mathrm{GeV}$

$\Gamma_{h}=4.07 \mathrm{MeV}$

$m_{t}=173.2 \mathrm{GeV}$

$\Gamma_{t}=0$

$\mu=\mu_{R}=\mu_{F}=\frac{1}{2}\left(E_{\mathrm{T}, t}+E_{\mathrm{T}, \bar{t}}\right)$,

where $E_{\mathrm{T}, t / \bar{t}}$ denotes the transverse energy of the top/antitop. In the predictions for top-pair plus jet production we recombine collinear photon-quark pairs within a cone of $R_{\gamma q}<0.1$ and cluster jets according to the anti- $k_{\mathrm{T}}$ algorithm implemented in FASTJET [87] and require

$p_{T, j}>30 \mathrm{GeV}$, and $\left|\eta_{j}\right|<4.5$.

Jets with a photonic energy fraction larger than $z_{\text {thr }}=0.5$ are discarded. No phase space cuts are applied to the final state top quarks.

We work in the five-flavour scheme and use the NNPDF3.0nnlo PDF set [89] with $\alpha_{\mathrm{s}}=0.118$ interfaced through LHAPDF6 [90]. The initial state QED evolution we thereby neglect has been shown to be negligible for $t \bar{t}$ production [8]. Here we present results for the LHC at a centreof-mass energy of $13 \mathrm{TeV}$. The relative electroweak corrections are fairly insensitive to the choices of $\mu_{R}$ and $\mu_{F}$. They slightly depend on the centre-of-mass energy: for larger energies the relative contribution of the $g g$ channel, which receives smaller EW corrections, increases with respect to the $q \bar{q}$ channel. Thus, the relative EW corrections are slightly (at the percent level) larger at $8 \mathrm{TeV}$ compared to $13 \mathrm{TeV}$, see also [5].

In Fig. 1 we show the NLO EW corrections together with the effect of the subleading Born and one-loop contributions to the top quark transverse momentum and the $t \bar{t}$ invariant mass in $t \bar{t}$ and $t \bar{t}+$ jet production. We find that the NLO EW corrections exhibit the expected electroweak Sudakov-like shape. They are small at low transverse momenta and invariant masses and continuously grow to reach about $-10(20) \%$ at a top-quark transverse momentum of $1(2) \mathrm{TeV}$ and about $-5(10) \%$ at $t \bar{t}$ invariant masses of $2(4) \mathrm{TeV}$, respectively. The NLO EW corrections to the $t \bar{t}+$ jet process reproduce those to the inclusive $t \bar{t}$ process to very high accuracy in these observables. Their ratio never exceeding a few percent. Thus, for the considered inclusive observables the electroweak corrections factorise to a very good approximation with respect to additional jet activity. This finding supports a multiplicative combination of QCD and NLO EW correc- 


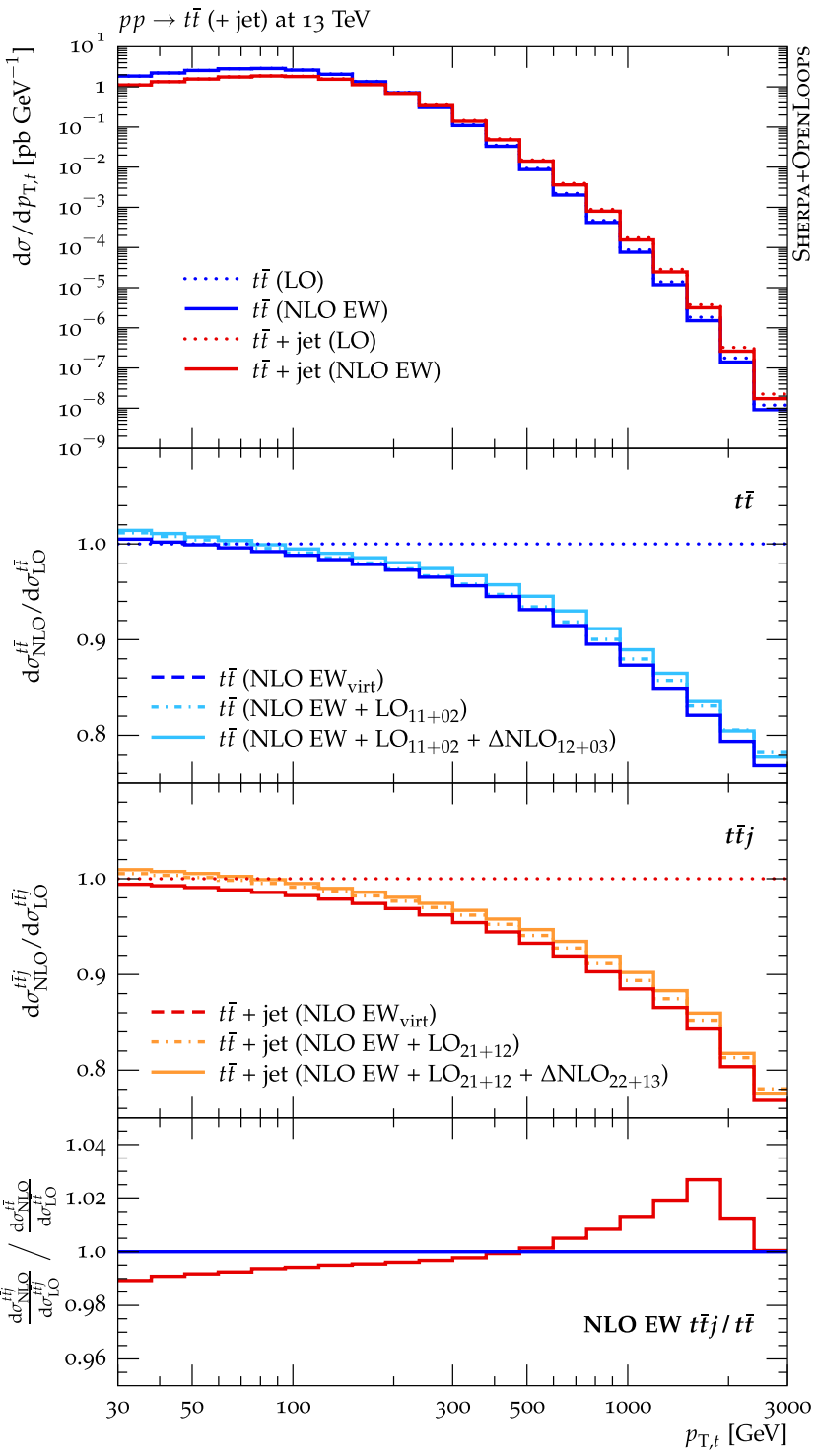

Fig. 1 Top quark transverse momentum (left) and top-antitop invariant mass (right) in inclusive $t \bar{t}$ production (blue) and $t \bar{t}+$ jet production (red) at NLO EW at $13 \mathrm{TeV}$ at the LHC. In $t \bar{t}+$ jet we require $p_{\mathrm{T}}>30 \mathrm{GeV}$. The top panel shows the differential cross section, while the three lower panels show, from top to bottom, the subleading Born and higher-order corrections to inclusive $t \bar{t}$ production and $t \bar{t}+$ jet production, respectively. Subleading Born and one-loop contributions are

tions in inclusive $t \bar{t}$ production. However, for less inclusive observables, for example due to requirements on additional hard jet activity, such a factorization of QCD and EW effects is less motivated.

In Fig. 1 subleading Born and one-loop contributions are shown with lighter shades of the colour of the respective processes, dash-dotted lines containing only the subleading Born contributions and solid lines containing all subleading Born and one-loop contributions. The subleading Born and one-loop orders contribute only at the percent level to the transverse momentum distribution, both for $t \bar{t}$ and $t \bar{t}+$ jet

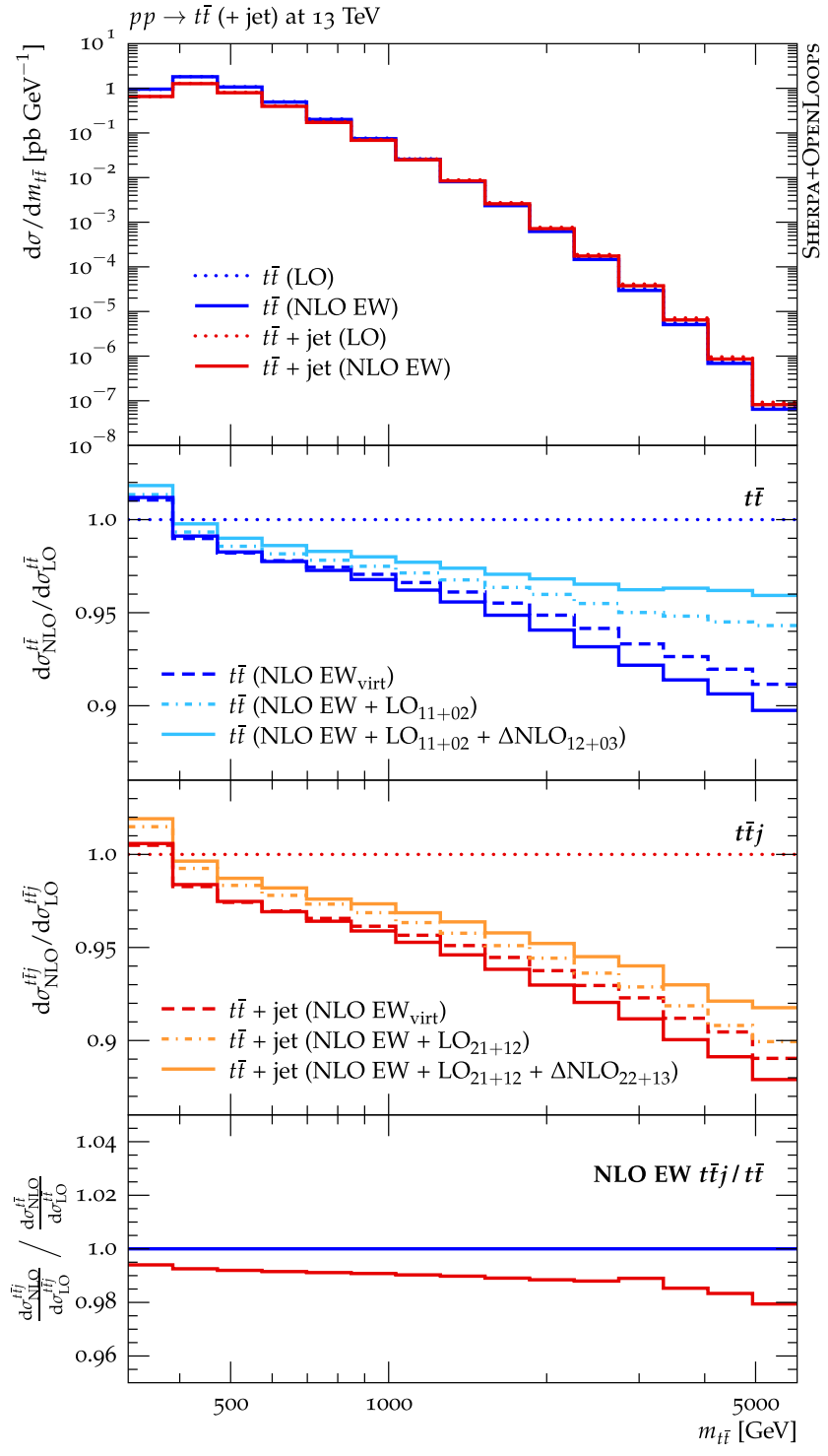

shown with lighter shades of the colour of the respective processes, dashed lines containing only the subleading Born contributions and solid lines containing all subleading Born and one-loop contributions. The lowest panel shows the ratio of the NLO EW corrections to the two processes. Corrections based on the NLO EW virt approximation are shown as the dashed line of the same colour as the exact NLO EW result

production. In particular, while for both $t \bar{t}$ and $t \bar{t}+$ jet the subleading Born contributions ( $\mathrm{LO}_{11}$ and $\mathrm{LO}_{21}$, respectively) are negligible, the inclusion of the sub-subleading Born contributions ( $\mathrm{LO}_{02}$ and $\mathrm{LO}_{12}$, respectively) increase the cross section an almost constant $\approx 1 \%$. On the other hand, for the invariant mass of the $t \bar{t}$-pair these subleading Born contributions show a different behaviour for inclusive $t \bar{t}$ production and $t \bar{t}$ production in association with a jet. While for the latter we again observe an almost constant increase of the cross section by $1-2 \%$, there is a clear shape distortion induced in the case of inclusive $t \bar{t}$ production. Here, for $m_{t \bar{t}}>2 \mathrm{TeV}$ 
the subleading Born contributions, largely dominated by the $\mathrm{LO}_{02}$ contribution, amount to $\approx \frac{1}{2}$ the NLO EW correction, only with opposite sign. The resulting compensation needs to be accounted for. Here, the subleading one-loop corrections are dominated by the $\Delta \mathrm{NLO}_{12}$ contributions and can in some sense be understood as the NLO QCD corrections to the sub-subleading Born $\mathrm{LO}_{02}$. For the case of barj production, here we want to note that the $\mathcal{O}\left(\alpha_{\mathrm{s}}^{2} \alpha^{2}\right)$ bremsstrahlung also comprises $t t V$ production with $V \rightarrow q \bar{q}$ decays, where $V=\left\{W^{ \pm}, Z\right\}$. Thus, in principle care has to be taken when such processes are considered as separate backgrounds in BSM searches. However, these subleading one-loop corrections contribute only at the percent level, with an increasing effect at very large $m_{t \bar{t}}$.

In Fig. 1 we also investigate the quality of the so-called $\mathrm{EW}_{\text {virt }}$ approximation [64] defined as

$$
\begin{aligned}
\mathrm{d} \sigma^{\mathrm{NLOEW}_{\text {virt }}=} & \mathrm{d} \Phi_{B}\left[\mathrm{~B}_{(n+2) 0}\left(\Phi_{B}\right)+\mathrm{V}_{(n+2) 1}\left(\Phi_{B}\right)\right. \\
& \left.+\int_{1} \mathrm{~d} \Phi_{1} \mathrm{R}_{(n+2) 1}^{\text {approx }}\left(\Phi_{B} \cdot \Phi_{1}\right)\right]
\end{aligned}
$$

where $n$ denotes the jet multiplicity in $t \bar{t}+n$ jet production. We define the approximated real-emission contribution $\mathrm{R}_{(n+2) 1}^{\mathrm{approx}}$ such that its integral over the real-emission phase space equals the standard Catani-Seymour I-operator. This approximation is both finite and local in the Born phase space and can hence be easily applied as a corrective weight in the multijet merging introduced in Sect. 3. By construction, it is expected to correctly reproduce the exact NLO EW corrections in the Sudakov limit, but also contain important nonlogarithmic terms extending its validity in practice.

In Fig. 1 the result using this approximation is detailed as the dashed line of the same colour as the exact NLO EW result. We find generally very good agreement, especially for the transverse momenta of the top quark in both $t \bar{t}$ and $t \bar{t}+$ jet production. Small differences are found for the invariant mass of the $t \bar{t}$-pair at values larger than $1 \mathrm{TeV}$, growing to relative difference of $1 \%$ at $5 \mathrm{TeV}$.

Finally, Fig. 2 details the higher-order EW corrections to the leading jet transverse momentum in $t \bar{t}+$ jet production. Similar but smaller corrections as in the top transverse momentum distribution are observed. In particular, we find NLO EW corrections of about $-10 \%$ at $2 \mathrm{TeV}$. Subleading Born and one-loop corrections are marginally relevant, i.e. they contribute below one percent. Again, we observe a very

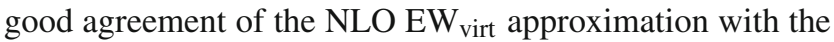
exact NLO EW result.

\section{MEPS@NLO QCD + EW}

To illustrate how electroweak corrections can easily be embedded in the multijet merging techniques used in the

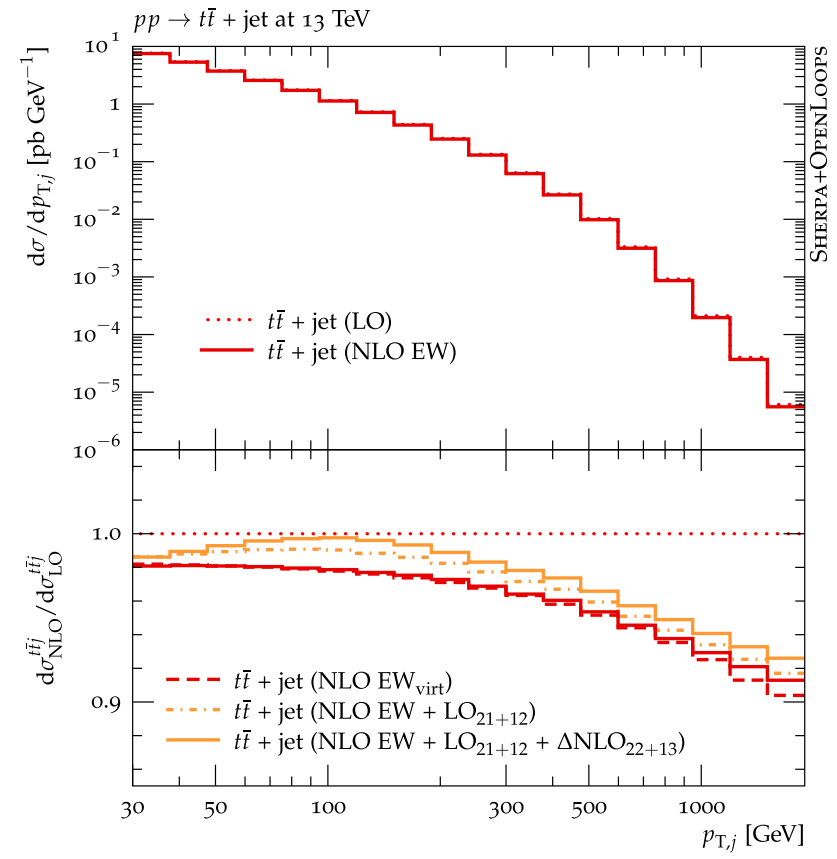

Fig. 2 Leading jet transverse momentum in $t \bar{t}+$ jet production at NLO EW at $13 \mathrm{TeV}$ at the LHC. The top panel shows the differential cross section, while the lower panel shows the subleading Born and one-loop electroweak corrections. Colour and line coding as in Fig. 1

SHERPA Monte Carlo event generator, we start with a schematic review of the MEPS method [41-43,91-94]. The aim is to generate inclusive event samples with a variable jet multiplicity, wherein the hardest $n=0,1, \ldots, n_{\max }$ jets, according to a measure $Q_{n}$, are described by the respective $n$-jet matrix elements at LO or NLO accuracy. A resolution criterion, $Q_{\text {cut }}$, is introduced to separate the $n$-jet state from the $n+1$-jet state. Thus, in every matrix element $Q_{1}>\cdots>Q_{n}>Q_{\text {cut }}$ holds.

In the leading order formulation of this merging method [41], MePs@Lo, the exclusive cross section with exactly $n<n_{\max }$ jets reads

$\mathrm{d} \sigma_{n}^{\mathrm{MEPs} @ \mathrm{LO}}=\mathrm{d} \Phi_{n} \mathrm{~B}_{n}\left(\Phi_{n}\right) \Theta\left(Q_{n}-Q_{\text {cut }}\right) \mathcal{F}_{n}\left(\mu_{Q}^{2} ;<Q_{\text {cut }}\right)$.

As in Sect. 2, $\mathrm{B}_{n}$ is the relevant Born matrix element including all PDF and symmetry/averaging factors, and $\Phi_{n}$ is the $n$-jet phase space configuration. Throughout, only the LO contribution, $\mathcal{O}\left(\alpha_{\mathrm{s}}^{2+n}\right)$ for $t \bar{t}+n$ jet production, are considered. The $\Theta$-function ensures that all jets are resolved. Finally, the parton shower generating functional $\mathcal{F}_{n}\left(\mu_{Q}^{2} ;<Q_{\text {cut }}\right)$ applies a truncated vetoed parton shower to the $n$-jet configuration, ensuring that all emissions fall into the unresolved region, $Q<Q_{\text {cut }}$. For the highest mulitplicity process, $n=n_{\max }$, the veto is relaxed to $Q_{n_{\max }}$ to arrive at a fully inclusive description. Through its veto it also applies a Sudakov form factor weight to the $n$-jet configuration, resum- 
ming the hierarchy of reconstructed parton shower branchings $\mu_{Q}^{2}=t_{0}, t_{1}, \ldots, t_{n}$. Together with the CKKW scale choice, $\mu_{R}=\mu_{\mathrm{CKKW}}$, defined through $[91,95]$

$\alpha_{\mathrm{s}}^{2+n}\left(\mu_{\mathrm{CKKW}}^{2}\right)=\alpha_{\mathrm{s}}^{2}\left(\mu_{\text {core }}^{2}\right) \cdot \alpha_{\mathrm{s}}\left(t_{1}\right) \ldots \alpha_{\mathrm{s}}\left(t_{n}\right)$,

and $\mu_{F}=\mu_{Q}=\mu_{\text {core }}$ a smooth transition across $Q_{\text {cut }}$ is ensured. The core scale is chosen as $[37,38]$

$\mu_{\text {core }}=\frac{1}{2}\left(\frac{1}{\hat{s}}+\frac{1}{m_{t}^{2}-\hat{t}}+\frac{1}{m_{t}^{2}-\hat{u}}\right)^{-\frac{1}{2}}$.

on the reconstructed core $2 \rightarrow 2$ process.

When upgrading the theoretical accuracy of the input $n$ jet cross section to NLO QCD accuracy one arrives at the MEPs@NLomethod $[42,43]$. Its exclusive $n$-jet cross sections, with $n<n_{\max }^{\mathrm{NLO}}$, are defined as

$$
\begin{aligned}
& \mathrm{d} \sigma_{n}^{\mathrm{MEPs} @ \mathrm{NLO}}=\left[\mathrm{d} \Phi_{n} \overline{\mathrm{B}}_{n}\left(\Phi_{n}\right) \overline{\mathcal{F}}_{n}\left(\mu_{Q}^{2} ;<Q_{\text {cut }}\right)\right. \\
& \left.\quad+\mathrm{d} \Phi_{n+1} \mathrm{H}_{n}\left(\Phi_{n+1}\right) \Theta\left(Q_{\text {cut }}-Q_{n+1}\right) \mathcal{F}_{n+1}\left(\mu_{Q}^{2} ;<Q_{\text {cut }}\right)\right] \\
& \quad \times \Theta\left(Q_{n}-Q_{\text {cut }}\right),
\end{aligned}
$$

based on the Mc@NLOexpressions of [96-98]. Therein, the $\overline{\mathrm{B}}_{n}$ term are the so-called MC@NLO standard events describing the production of $n$ resolved partons with $Q_{n}>Q_{\text {cut }}$ at matrix-element level including virtual corrections. The $(n+1)$-th emission is generated through a fully colour- and spin-correlated one-step parton shower $\overline{\mathcal{F}}_{n}$ [96], restricted to the unresolved region. The $\overline{\mathrm{B}}_{n}$ function takes the form

$$
\begin{aligned}
\overline{\mathrm{B}}_{n}\left(\Phi_{n}\right)= & \mathrm{B}_{n}\left(\Phi_{n}\right)+\tilde{\mathrm{V}}_{n}\left(\Phi_{n}\right) \\
& +\int \mathrm{d} \Phi_{1} \mathrm{D}_{n}\left(\Phi_{n}, \Phi_{1}\right) \Theta\left(\mu_{Q}^{2}-t_{n+1}\right) .
\end{aligned}
$$

wherein $\tilde{\mathrm{V}}_{n}\left(\Phi_{n}\right)$ consisting of virtual QCD corrections and initial-state collinear mass-factorisation counterterms and $\mathrm{D}_{n}$ is the evolution kernel of $\overline{\mathcal{F}}_{n}$ [42] and the $\Theta$-function restricts the parton shower phase space. The $\mathrm{H}_{n}$ term, on the other hand, corresponds to so-called Mc@NLO hard events. Its purpose is to correct the approximate emission pattern of the $\overline{\mathcal{F}}_{n}$ resummation kernels and guarantee NLO QCD accuracy. It is thus also subject to the $\left(Q_{n+1}<Q_{\text {cut }}\right)$ requirement. Again, for $n=n_{\max }^{\mathrm{NLO}}=n_{\max }$, the requirement on the $(n+1)$-th emission is relaxed to $Q_{n_{\max }}$. The $\mathrm{H}_{n}$ function takes the form

$$
\mathrm{H}_{n}\left(\Phi_{n+1}\right)=\mathrm{R}_{n}\left(\Phi_{n+1}\right)-\mathrm{D}_{n}\left(\Phi_{n+1}\right) \Theta\left(\mu_{Q}^{2}-t_{n+1}\right),
$$

wherein $\mathrm{R}_{n}\left(\Phi_{n+1}\right)$ denotes the real-emission matrix elements.

It is possible to set $n_{\max }^{\mathrm{NLO}}<n_{\max }$, i.e. describing only the $n_{\max }^{\mathrm{NLO}}$ lowest jet multiplicities at NLO QCD accuracy and adding the next $n_{\max }-n_{\max }^{\mathrm{NLO}}$ jet multiplicities at leading order. The resulting MENLOPS method [43,94,99] use Eq. (3.4) to describe the multiplicities described at NLO QCD. The subsequent LO multiplicities with $n=n_{\max }^{\mathrm{NLO}}+k(k>0)$ are defined through

$$
\begin{aligned}
\mathrm{d} \sigma_{n}^{(\mathrm{MENLOPS})}= & \mathrm{d} \Phi_{n} k_{n_{\max }^{\mathrm{NLO}}}\left(\Phi_{n_{\max }^{\mathrm{NLO}}}\left(\Phi_{n}\right)\right) \mathrm{B}_{n}\left(\Phi_{n}\right) \\
& \times \Theta\left(Q_{n}-Q_{\text {cut }}\right) \mathcal{F}_{n}\left(\mu_{Q}^{2} ;<Q_{\text {cut }}\right)
\end{aligned}
$$

i.e. it supplies the MEPs@Lo expression of Eq. (3.1)with the local $K$-factor, defined on the highest multiplicity described at NLO QCD,

$$
k_{n}\left(\Phi_{n}\right)=\frac{\overline{\mathrm{B}}_{n}\left(\Phi_{n}\right)}{\mathrm{B}_{n}\left(\Phi_{n}\right)}\left(1-\frac{\mathrm{H}_{n}\left(\Phi_{n+1}\right)}{\mathrm{B}_{n+1}\left(\Phi_{n+1}\right)}\right)+\frac{\mathrm{H}_{n}\left(\Phi_{n+1}\right)}{\mathrm{B}_{n+1}\left(\Phi_{n+1}\right)}
$$

This expression expands to $\mathcal{O}\left(\alpha_{\mathrm{s}}\right)$ such that the leading order accuracy of the $n$-jet sample is not affected. Here $\Phi_{n_{\max }^{\mathrm{NLO}}}\left(\Phi_{n}\right)$ denotes the projection of the $n$-jet phase space on the $n_{\max }^{\mathrm{NLO}}$ jet one, taken from the identified cluster history constructed during the identification of the emission scales $t_{i}$ [41]. This $K$-factor is constructed such that the combination of the exclusive $n_{\max }^{\mathrm{NLO}}$-jet process at NLO QCD and the inclusive $\left(n_{\max }^{\mathrm{NLO}}+1\right)$-jet process at LO reproduces the inclusive $n_{\max }^{\mathrm{NLO}}-$ jet process at NLO QCD exactly and, thus, minimises discontinuities across $Q_{\text {cut }}$. The inclusion of additional jet multiplicities at LO is in particular relevant for the reliable modelling of $t \bar{t}+$ multijet signatures in BSM background simulations.

In order to incorporate approximate NLO EW corrections in the MEPs@NLOframework we replace the usual NLO QCD $\bar{B}_{n}$ function of Eq. (3.5) with [64]

$$
\begin{aligned}
\overline{\mathrm{B}}_{n, \mathrm{QCD}+\mathrm{EW}}\left(\Phi_{n}\right)= & \overline{\mathrm{B}}_{n}\left(\Phi_{n}\right)+\mathrm{V}_{n, \mathrm{EW}}\left(\Phi_{n}\right) \\
& +\mathrm{I}_{n, \mathrm{EW}}\left(\Phi_{n}\right)+\mathrm{B}_{n, \mathrm{sub}}\left(\Phi_{n}\right) .
\end{aligned}
$$

incorporating NLO EW corrections in the $\mathrm{EW}_{\text {virt }}$ approximation defined in Eq. (2.6), together with subleading Born contributions $\mathrm{B}_{n, \text { sub }}$. Again, $\mathrm{V}_{n, \mathrm{EW}}\left(\Phi_{n}\right)$ and $\mathrm{I}_{n, \mathrm{EW}}\left(\Phi_{n}\right)$ represent the renormalised virtual corrections of $\mathcal{O}\left(\alpha_{\mathrm{s}}^{2+n} \alpha\right)$ and the approximate real emission corrections of the same order, integrated over the real emission phase space, in the form of the NLO EW generalisation of the Catani-Seymour I operator, respectively, as discussed in Sect. 2.

In the multijet merged setup, the real emission corrections as well as the additional LO multiplicities are supplied by COMIX [100] and then showered using the CsSHOWER [101] based on Catani-Seymour splitting kernels. In the following, we perform both a technical validation of the presented algorithm at parton-level and then compare the full particle-level 


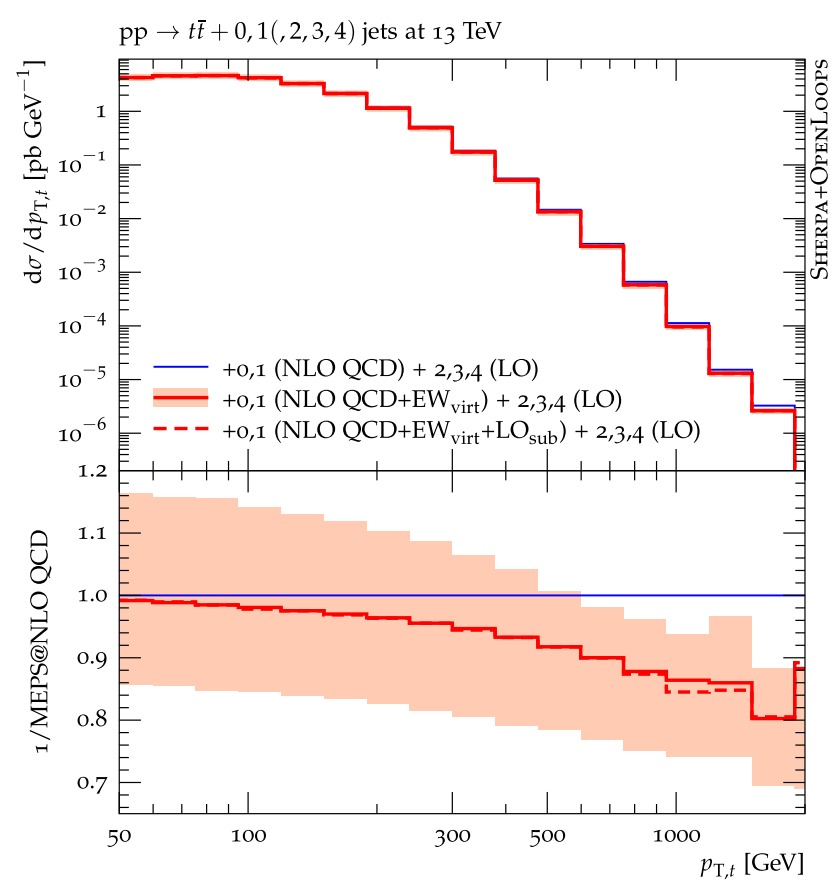

Fig. 3 Top-quark transverse momentum distribution (left) and topantitop invariant mass (right) at parton-level with stable tops for the LHC with 13 TeV. Compared are MEPs@NLOQCD and MEPs@NLOQCD

simulation including spin correlated LO top-quark decays against data.

\section{Results}

In Figs. 3 and 4 we present parton-level multi-jet merged MEPs@NLOQCD + EW virt predictions at the LHC with $13 \mathrm{TeV}$. Here the top quark is treated as stable and no nonperturbative effects are included. Input parameters and settings are chosen as detailed in Sect. 2. We merge $t \bar{t}$ plus zero and one jet production based on NLO matrix elements including $\mathcal{O}\left(\alpha_{\mathrm{S}}\right)$ QCD corrections and $\mathcal{O}(\alpha)$ EW corrections in the $\mathrm{EW}_{\text {virt }}$ approximation. In all merged predictions we choose $Q_{\text {cut }}=30 \mathrm{GeV} .{ }^{3}$ The effect of additionally including the subleading Born contributions of $\mathcal{O}\left(\alpha_{\mathrm{s}}^{1+n} \alpha\right)$ and $\mathcal{O}\left(\alpha_{\mathrm{s}}^{n} \alpha^{2}\right)$ in the merging is shown explicitly. In order to allow for a direct comparison with the corresponding fixed-order results we chose renormalisation and factorisation scales according to Eq. (2.4). The shown error bands indicate resulting factor2 QCD scale variations. In the ratio of the MEPs@ NLOQCD $+\mathrm{EW}_{\text {virt }}$ predictions over the MEPs@ NLOQCD predictions we recover EW correction factors consistent with the fixed-

3 In Appendix B of [64] it was shown that even for the prediction of observables in the multi- $\mathrm{TeV}$ regime the associated uncertainty related to variations of the merging scale are very small.

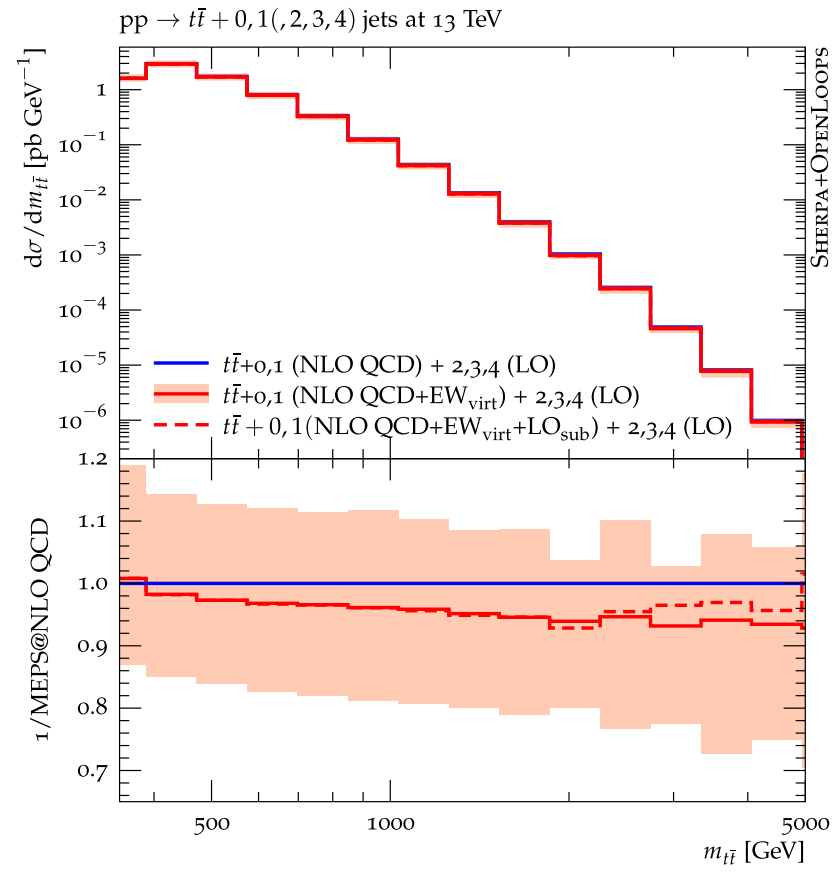

$+\mathrm{EW}_{\text {virt }}$ predictions and the effect of subleading Born contributions. Error bands are due to QCD scale variations

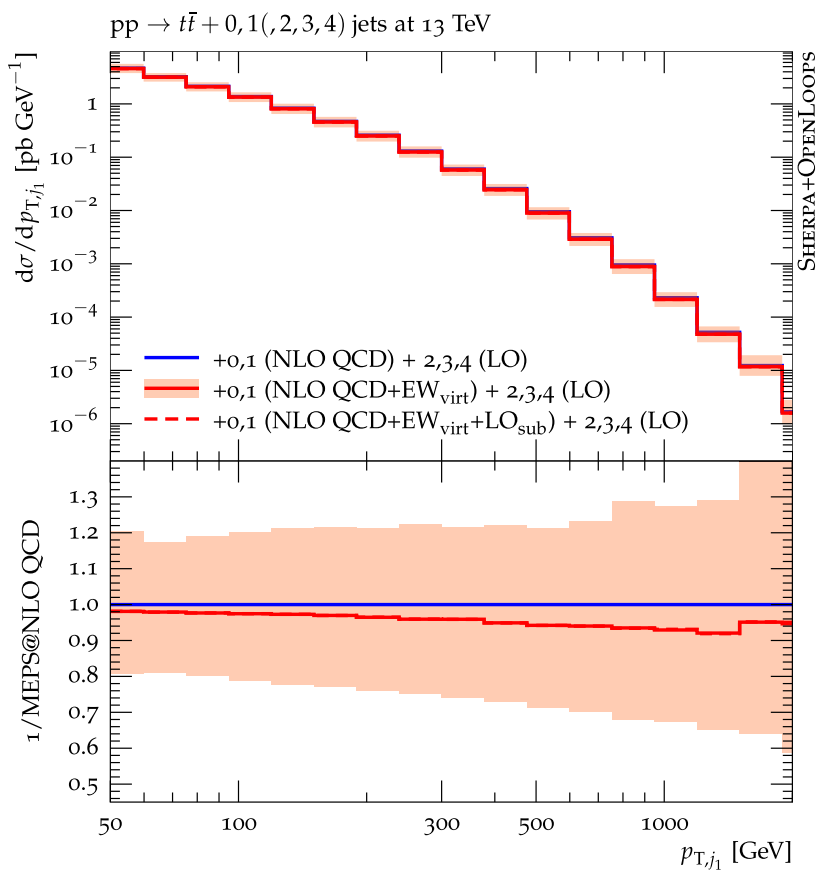

Fig. 4 Leading jet transverse momentum distribution at the LHC with 13 TeV comparing MEPs@ NLO QCD and MEPs@ NLOQCD + EW virt parton-level predictions. Error bands are due to QCD scale variations

order results presented in Sect. 2. The same also holds for the effect of the subleading Born contributions. 


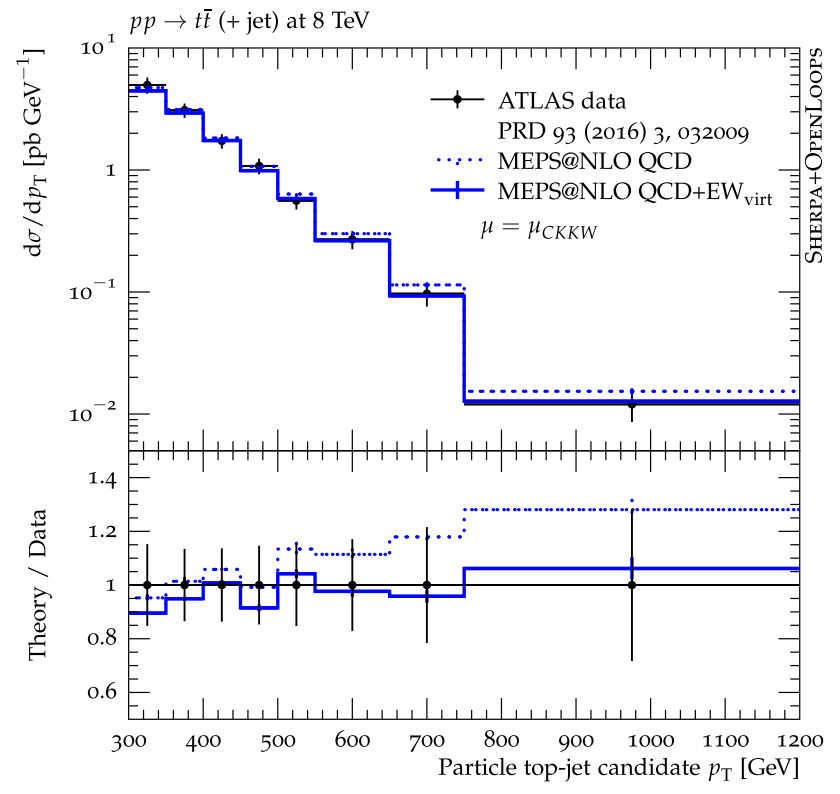

Fig. 5 Comparison of the MEPS@NLO QCD and MEPS@NLO QCD $+\mathrm{EW}_{\text {virt }}$ predictions for the transverse momentum distribution of hadronically decaying top candidates against an $8 \mathrm{TeV}$ ATLAS measurement in the lepton + jets channel based on a boosted top selection [59]

Finally, in Fig. 5 we present full particle-level MEPs@NLOQCD + EW virt predictions for multijet-merged top-pair production including spin-correlated top quark decays [102] in the semileptonic decay channel. Here, also non-perturbative effects due to multiple interaction simulation [103], hadronisation [104] and hadron decays, as well as higher-order QED effects included through the soft-photon resummation of [65] have been included. These predictions are compared to experimental data taken by the ATLAS experiment [59] at the $\mathrm{LHC}$ at $8 \mathrm{TeV}$ measuring the transverse momentum distribution of reconstructed top-quark candidates. The corresponding analysis is implemented in RIVET [105] and entails a reconstruction of the transverse momentum of the hadronically decaying top-quark candidates with $p_{\mathrm{T}}>300 \mathrm{GeV}$. In this measurement, the boosted top-quark candidate is identified as a single large-radius jet $(R=1.0)$ using jet substructure techniques.

We find a significant improvement of the agreement between MC simulation and data when electroweak corrections are included, although the statistical prowess of the data sample as well as the high- $p_{\mathrm{T}}$ reach are limited in this measurement.

\section{Conclusions}

In this paper we have presented the first predictions for toppair plus jet production including Born and one-loop EW corrections. We compared these corrections with the ones for top-pair production and overall found a universal behaviour indicating a factorisation of the EW corrections with respect to additional jet radiation for inclusive observables. Subsequently, based on the MEPs@NLOmultijet merging framework in SHERPA combined with OPENLOOPS, we derived parton- and particle-level predictions for inclusive top-pair production including NLO QCD and EW corrections. The EW corrections are incorporated in an approximation, based on exact virtual NLO EW contributions combined with integrated-out QED bremsstrahlung. We showed that this approximation is able to reproduce the full NLO EW result for $t \bar{t}$ and $t \bar{t}+$ jet production at the percent level. Comparing our predictions against a recent measurement for the topquark $p_{\mathrm{T}}$-spectrum performed by ATLAS in the lepton + jet channel we find very good agreement between the NLO multi-jet merged Monte Carlo predictions and data when the EW corrections are included.

Acknowledgements We thank Frank Krauss and Stefano Pozzorini for useful conversations. The latter we also thank for valuable comments on the manuscript. This work has received funding from the European Union's Horizon 2020 research and innovation programme as part of the Marie Skodowska-Curie Innovative Training Network MCnetITN3 (Grant Agreement no. 722104).

Open Access This article is distributed under the terms of the Creative Commons Attribution 4.0 International License (http://creativecomm ons.org/licenses/by/4.0/), which permits unrestricted use, distribution, and reproduction in any medium, provided you give appropriate credit to the original author(s) and the source, provide a link to the Creative Commons license, and indicate if changes were made. Funded by SCOAP ${ }^{3}$.

\section{References}

1. M. Czakon, P. Fiedler, A. Mitov, Phys. Rev. Lett. 110, 252004 (2013). arXiv:1303.6254

2. M. Czakon, D. Heymes, A. Mitov, Phys. Rev. Lett. 116(8), 082003 (2016). arXiv: 1511.00549

3. J.H. Kühn, A. Scharf, P. Uwer, Eur. Phys. J. C 51, 37-53 (2007). arXiv:hep-ph/0610335

4. W. Bernreuther, M. Fuecker, Z.-G. Si, Phys. Rev. D 74, 113005 (2006). arXiv:hep-ph/0610334

5. J.H. Kühn, A. Scharf, P. Uwer, Phys. Rev. D 91(1), 014020 (2015). arXiv: 1305.5773

6. W. Bernreuther, Z.-G. Si, Nucl. Phys. B 837, 90-121 (2010). arXiv: 1003.3926

7. W. Hollik, D. Pagani, Phys. Rev. D 84, 093003 (2011). arXiv: 1107.2606

8. D. Pagani, I. Tsinikos, M. Zaro, Eur. Phys. J. C 76(9), 479 (2016). arXiv: 1606.01915

9. M. Czakon, D. Heymes, A. Mitov, D. Pagani, I. Tsinikos, M. Zaro, JHEP 10, 186 (2017). arXiv: 1705.04105

10. M. Czakon, D. Heymes, A. Mitov, D. Pagani, I. Tsinikos, M. Zaro. arXiv: 1711.03945

11. S. Dittmaier, P. Uwer, S. Weinzierl, Phys. Rev. Lett. 98, 262002 (2007). arXiv:hep-ph/0703120 
12. K. Melnikov, M. Schulze, Nucl. Phys. B 840, 129-159 (2010). arXiv: 1004.3284

13. A. Bredenstein, A. Denner, S. Dittmaier, S. Pozzorini, Phys. Rev. Lett. 103, 012002 (2009). arXiv:0905.0110

14. A. Bredenstein, A. Denner, S. Dittmaier, JHEP 03, 021 (2010). arXiv: 1001.4006

15. G. Bevilacqua, M. Czakon, C.G. Papadopoulos, R. Pittau, M. Worek, JHEP 09, 109 (2009). arXiv:0907.4723

16. G. Bevilacqua, M. Czakon, C.G. Papadopoulos, M. Worek, Phys. Rev. Lett. 104, 162002 (2010). arXiv: 1002.4009

17. G. Bevilacqua, M. Czakon, C.G. Papadopoulos, M. Worek, Phys. Rev. D 84, 114017 (2011). arXiv:1108.2851

18. S. Höche, P. Maierhöfer, N. Moretti, S. Pozzorini, E. Siegert, Eur. Phys. J. C 77(3), 145 (2017). arXiv:1607.06934

19. K. Melnikov, M. Schulze, JHEP 08, 049 (2009). arXiv:0907.3090

20. J.M. Campbell, R.K. Ellis, J. Phys. G 42(1), 015005 (2015). arXiv: 1204.1513

21. G. Bevilacqua, M. Czakon, A. van Hameren, C.G. Papadopoulos, JHEP 02, 083 (2011). arXiv:1012.4230

22. A. Denner, S. Dittmaier, S. Kallweit, S. Pozzorini, Phys. Rev. Lett. 106, 052001 (2011). arXiv:1012.3975

23. A. Denner, S. Dittmaier, S. Kallweit, S. Pozzorini, JHEP 10, 110 (2012). arXiv:1207.5018

24. F. Cascioli, S. Kallweit, P. Maierhöfer, S. Pozzorini, Eur. Phys. J. C 74(3), 2783 (2014). arXiv: 1312.0546

25. R. Frederix, Phys. Rev. Lett. 112(8), 082002 (2014). arXiv: 1311.4893

26. A. Denner, M. Pellen, JHEP 02, 013 (2018). arXiv:1711.10359

27. A. Denner, M. Pellen, JHEP 08, 155 (2016). arXiv:1607.05571

28. K. Melnikov, A. Scharf, M. Schulze, Phys. Rev. D 85, 054002 (2012). arXiv:1111.4991

29. G. Bevilacqua, H.B. Hartanto, M. Kraus, M. Worek, Phys. Rev. Lett. 116(5), 052003 (2016). arXiv: 1509.09242

30. S. Frixione, P. Nason, B.R. Webber, JHEP 08, 007 (2003). arXiv:hep-ph/0305252

31. S. Frixione, P. Nason, G. Ridolfi, JHEP 09, 126 (2007). arXiv:0707.3088

32. A. Kardos, C. Papadopoulos, Z. Trocsanyi, Phys. Lett. B 705, 76-81 (2011). arXiv:1101.2672

33. S. Alioli, S.-O. Moch, P. Uwer, JHEP 01, 137 (2012). arXiv: 1110.5251

34. M. Czakon, H.B. Hartanto, M. Kraus, M. Uwer, JHEP 06, 033 (2015). arXiv: 1502.00925

35. A. Kardos, Z. Trocsanyi, J. Phys. G 41, 075005 (2014). arXiv: 1303.6291

36. F. Cascioli, P. Maierhöfer, N. Moretti, S. Pozzorini, F. Siegert, Phys. Lett. B 734, 210-214 (2014). arXiv:1309.5912

37. S. Höche, J. Huang, G. Luisoni, M. Schönherr, J. Winter, Phys. Rev. D 88(1), 014040 (2013). arXiv:1306.2703

38. S. Höche, F. Krauss, P. Maierhöfer, S. Pozzorini, M. Schönherr, F. Siegert, Phys. Lett. B 748, 74-78 (2015). arXiv:1402.6293

39. G. Bevilacqua, M.V. Garzelli, A. Kardos. arXiv:1709.06915

40. T. Jezo, J.M. Lindert, N. Moretti, S. Pozzorini. arXiv:1802.00426

41. S. Höche, F. Krauss, S. Schumann, F. Siegert, JHEP 05, 053 (2009). arXiv:0903.1219

42. S. Höche, F. Krauss, M. Schönherr, F. Siegert, JHEP 04, 027 (2013). arXiv:1207.5030

43. T. Gehrmann, S. Hoche, F. Krauss, M. Schönherr, F. Siegert, JHEP 01, 144 (2013). arXiv:1207.5031

44. J.M. Campbell, R.K. Ellis, P. Nason, E. Re, JHEP 04, 114 (2015). arXiv: 1412.1828

45. T. Jezo, J.M. Lindert, P. Nason, C. Oleari, S. Pozzorini, Eur. Phys. J. C 76(12), 691 (2016). arXiv:1607.04538

46. T. Jezo, P. Nason, JHEP 12, 065 (2015). arXiv:1509.09071

47. CMS Collaboration, S. Chatrchyan et al., JHEP 11, 067 (2012). arXiv:1208.2671
48. CMS Collaboration, S. Chatrchyan et al., JHEP 02, 024 (2014). arXiv:1312.7582. [Erratum: JHEP 02, 102 (2014)]

49. ATLAS Collaboration, G. Aad et al., Eur. Phys. J. C 74(10), 3109 (2014). arXiv:1406.5375. [Addendum: Eur. Phys. J. C 76, no. 11, 642 (2016)]

50. CMS Collaboration, V. Khachatryan et al., Phys. Rev. Lett. 116(5), 052002 (2016). arXiv: 1510.05302

51. ATLAS Collaboration, M. Aaboud et al., Phys. Lett. B 761, 136157 (2016). arXiv:1606.02699. [Erratum: Phys. Lett. B 772, 879 (2017)]

52. ATLAS, CMS Collaboration, A.K. Nayak, PoS CKM 2016, 117 (2017)

53. M. Cacciari, M. Czakon, M. Mangano, A. Mitov, P. Nason, Phys. Lett. B 710, 612-622 (2012). arXiv: 1111.5869

54. M. Beneke, P. Falgari, S. Klein, C. Schwinn, Nucl. Phys. B 855, 695-741 (2012). arXiv:1109.1536

55. CMS Collaboration, S. Chatrchyan et al., Eur. Phys. J. C 73(3), 2339 (2013). arXiv:1211.2220

56. ATLAS Collaboration, G. Aad et al., Phys. Rev. D 90(7), 072004 (2014). arXiv:1407.0371

57. ATLAS Collaboration, G. Aad et al., JHEP. 06, 100 (2015). arXiv: 1502.05923

58. CMS Collaboration, V. Khachatryan et al., Eur. Phys. J. C 75(11), 542 (2015). arXiv: 1505.04480

59. ATLAS Collaboration, G. Aad et al., Phys. Rev. D 93(3), 032009 (2016). arXiv: 1510.03818

60. CMS Collaboration, V. Khachatryan et al., Phys. Rev. D 95(9), 092001 (2017). arXiv:1610.04191

61. CMS Collaboration, A.M. Sirunyan et al. arXiv:1708.07638

62. ATLAS Collaboration, M. Aaboud et al., Eur. Phys. J. C 77(5), 292 (2017). arXiv:1612.05220

63. ATLAS Collaboration, M. Aaboud et al. arXiv:1801.02052

64. S. Kallweit, J.M. Lindert, P. Maierhöfer, S. Pozzorini, M. Schönherr, JHEP 04, 021 (2016). arXiv: 1511.08692

65. M. Schönherr, F. Krauss, JHEP 12(1), 018 (2008). arXiv:0810.5071

66. A.V. Manohar, P. Nason, G.P. Salam, G. Zanderighi, JHEP 12, 046 (2017). arXiv: 1708.01256

67. A. Denner, S. Pozzorini, Eur. Phys. J. C 18, 461-480 (2001). arXiv:hep-ph/0010201

68. A. Denner, S. Pozzorini, Eur. Phys. J. C 21, $63-79$ (2001) arXiv:hep-ph/0104127

69. A. Denner, B. Jantzen, S. Pozzorini, JHEP 11, 062 (2008). arXiv:0809.0800

70. F. Cascioli, P. Maierhöfer, S. Pozzorini, Phys. Rev. Lett. 108, 111601 (2012). arXiv:1111.5206

71. The OPENLOOPS one-loop generator by F. Cascioli, J. Lindert, P. Maierhöfer and S. Pozzorini is publicly available at http:// openloops.hepforge.org

72. S. Kallweit, J.M. Lindert, P. Maierhöfer, S. Pozzorini, M. Schönherr, JHEP 04, 012 (2015). arXiv: 1412.5157

73. A. Denner, S. Dittmaier, L. Hofer, Comput. Phys. Commun. 212, 220-238 (2017). arXiv: 1604.06792

74. G. Ossola, C.G. Papadopoulos, R. Pittau, JHEP 03, 042 (2008). arXiv:0711.3596

75. F. Krauss, R. Kuhn, G. Soff, JHEP 02, 044 (2002). arXiv:hep-ph/0109036

76. S. Catani, M.H. Seymour, Nucl. Phys. B 485, 291-419 (1997). arXiv:hep-ph/9605323. [Erratum: Nucl. Phys. B 510, 503 (1998)]

77. S. Dittmaier, Nucl. Phys. B 565, 69-122 (2000). arXiv:hep-ph/9904440

78. S. Catani, S. Dittmaier, M.H. Seymour, Z. Trocsanyi, Nucl. Phys. B 627, 189-265 (2002). arXiv:hep-ph/0201036

79. T. Gleisberg, F. Krauss, Eur. Phys. J. C 53, 501-523 (2008). arXiv:0709.2881 
80. S. Dittmaier, A. Kabelschacht, T. Kasprzik, Nucl. Phys. B 800 , 146-189 (2008). arXiv:0802.1405

81. M. Schönherr Eur, Phys. J. C 78(2), 119 (2018). arXiv: 1712.07975

82. B. Biedermann, S. Bräuer, A. Denner, M. Pellen, S. Schumann, J.M. Thompson, Eur. Phys. J. C 77, 492 (2017). arXiv: 1704.05783

83. S. Kallweit, J.M. Lindert, S. Pozzorini, M. Schönherr, JHEP 11, 120 (2017). arXiv: 1705.00598

84. M. Chiesa, N. Greiner, M. Schönherr, F. Tramontano, JHEP 10, 181 (2017). arXiv: 1706.09022

85. N. Greiner, M. Schönherr, JHEP 01, 079 (2018). arXiv: 1710.11514

86. A. Denner, S. Dittmaier, M. Roth, L.H. Wieders, Nucl. Phys. B 724, 247-294 (2005). arXiv:hep-ph/0505042. [Erratum: Nucl. Phys. B 854, 504 (2012)]

87. M. Cacciari, G.P. Salam, G. Soyez, Eur. Phys. J. C 72, 1896 (2012). arXiv: 1111.6097

88. Particle Data Group Collaboration, K.A. Olive et al., Chin. Phys. C 38, 090001 (2014)

89. NNPDF Collaboration, R.D. Ball et al., JHEP 04, 040 (2015). arXiv: 1410.8849

90. A. Buckley, J. Ferrando, S. Lloyd, K. Nordström, B. Page, M. Rüfenacht, M. Schönherr, G. Watt, Eur. Phys. J. C 75, 132 (2015). arXiv: 1412.7420

91. S. Catani, F. Krauss, R. Kuhn, B.R. Webber, JHEP 11, 063 (2001). arXiv:hep-ph/0109231

92. L. Lönnblad, JHEP 05, 046 (2002). arXiv:hep-ph/0112284

93. E. Krauss, JHEP 08, 015 (2002). arXiv:hep-ph/0205283
94. S. Höche, F. Krauss, M. Schönherr, F. Siegert, JHEP 08, 123 (2011). arXiv:1009.1127

95. E. Bothmann, M. Schönherr, S. Schumann, Eur. Phys. J. C 76(11), 590 (2016). arXiv: 1606.08753

96. S. Höche, F. Krauss, M. Schönherr, Siegert F, JHEP 09, 049 (2012). arXiv:1111.1220

97. S. Höche, F. Krauss, M. Schönherr, F. Siegert, Phys. Rev. Lett. 110(5), 052001 (2013).arXiv:1201.5882

98. S. Höche, M. Schönherr, Phys. Rev. D 86, 094042 (2012). arXiv: 1208.2815

99. S. Höche, F. Krauss, S. Pozzorini, M. Schönherr, J.M. Thompson, K.C. Zapp, Phys. Rev. D 89(9), 093015 (2014). arXiv:1403.7516

100. T. Gleisberg, S. Höche, JHEP 12, 039 (2008). arXiv:0808.3674

101. S. Schumann, F. Krauss, JHEP 03, 038 (2008). arXiv:0709.1027

102. S. Höche, S. Kuttimalai, S. Schumann, F. Siegert, Eur. Phys. J. C 75(3), 135 (2015). arXiv: 1412.6478

103. A. De Roeck, H. Jung (eds.), HERA and the LHC: A Workshop on the Implications of HERA for LHC Physics: Proceedings Part A (CERN, Geneva, 2005)

104. J.-C. Winter, F. Krauss, G. Soff, Eur. Phys. J. C 36, 381-395 (2004). arXiv:hep-ph/0311085

105. A. Buckley, J. Butterworth, L. Lönnblad, D. Grellscheid, H. Hoeth, J. Monk, H. Schulz, F. Siegert, Comput. Phys. Commun. 184, 2803-2819 (2013). arXiv:1003.0694 